\title{
The impact of flooding on transport networks: optimization of action plans in a decision support tool
}

\author{
G. Morel $^{1} \&$ F. Hissel ${ }^{2}$ \\ ${ }^{1}$ Department of Urban Systems Engineering, AVENUES Research Group, \\ Compiègne University of Technology (UTC), France \\ ${ }^{2}$ Centre d'Etudes Techniques Maritimes et Fluviales, Laboratoire \\ Roberval (UTC), France
}

\begin{abstract}
The OSIRIS-Inondation tool was created as part of the OSIRIS FP5-IST project and is now used by many French towns to generate a flood action plan for crisis preparation and delivery of operational support to the emergency services during crisis management or training phases. At present, the action plan is calculated on the basis of accurate information on available human and material resources, crisis scenarios, key facilities and the actions needed to protect the latter, while taking account of the direct, local impact of the flooding. However, the ability to correctly implement an action plan has never been analysed - notably in the event when a flooding closes down transport networks and thus prevents access (in full or in part) to the key facilities in question. The goal of the ACCEL project (funded by the Picardie Region in northern France) is to develop a method for calculating the accessibility of predetermined facilities and to integrate it into the OSIRIS platform. In order to address these issues, we developed an approach for (i) identifying the data required to calculate accessibility, (ii) cross-checking these data against flood scenarios, (iii) determining which sections of transport networks will be disrupted and (iv) calculating optimal itineraries to key facilities. Validation of this methodology in a case study of the Compiègne urban area (using ArcGIS software) enabled the development of a self-contained route-planning module for integration into OSIRIS action plans.
\end{abstract}

Keywords: crisis management, accessibility, road disruption, emergency plans, decision support, systemic vulnerability. 


\section{Introduction}

In 2004, changes in France's safety regulations transferred part of the responsibility for crisis preparation and management to the country's communes and their mayors. However, most of these local authorities have few resources, lack preparation and information and thus are keenly awaiting tools to help them implement the regulations at a local level.

OSIRIS-Inondation (designed and tested as part of the European Unionfunded (FP5-IST) OSIRIS project) is a tool which precisely addresses this issue (see §2). It is now being used by several dozen French towns to elaborate flood scenarios and action plans (crisis preparation) and implement the latter as part of a real crisis or a training exercise (crisis management). In accordance with guidelines issued by the French Ministry of the Interior, the OSIRIS-Inondation system has been elaborated with and for field-based stakeholders (town councils, local emergency services, etc.) and has been funded, developed and deployed by the public river basin management authorities for the Loire (EPLoire) and the Meuse (EPAMA).

At present, the action plan generated by the OSIRIS platform during crisis preparation and then executed during crisis management takes account of the direct, local impact of flooding. Nevertheless, OSIRIS does not analyze how these actions should be performed optimally - particularly when flooding cuts or disrupts transport networks and thus partly or fully blocks access to strategic facilities. This is a recurrent problem for the emergency services. How can we access a given hospital when the flooding reaches a certain level? If there are several emergency service centres in town, which should be activated for rapid action if a particular school is affected by the hazard? Accessibility to strategic facilities [1] and evacuation of the population in crisis situations [2] are major issues in crisis preparation and management, as has been amply demonstrated by recent events (e.g. Cyclone Katrina in New Orleans, 2005).

In order to address these issues, the ACCEL project (funded by the Picardy Regional Council, France) has elaborated a methodology for (i) gathering the data needed to calculate accessibility, (ii) cross-relating these data with flood scenarios (in order to determine which sections of the transport network are disrupted) and (iii) providing the emergency services with the optimal itinerary for accessing the affected facilities as a function of the flooding situation (see $\S 3)$. This methodology was first validated for the Compiègne urban area (in the Picardie region of northern France) with ArcGIS software and then used to develop an accessibility calculation module that will be integrated into OSIRIS' action plans (see $\S 4$ ).

\section{OSIRIS-Inondation: a decision support tool for flood crisis planning and management}

\subsection{Decision support in crisis preparation}

During the crisis preparation phase, OSIRIS-Inondation provides a coherent methodological framework and a man-machine interface for facilitating the 
constitution of a Local Emergency Plan (LEP): identification of the flood risks to which the town is exposed, the key facilities and their level of vulnerability, the action to be taken and the requisite resources. This process ensures that (i) the main crisis scenarios have been thoroughly analyzed, (ii) the corresponding actions have been well planned and (iii) the validity and coherence of the action plans and corresponding route maps have been checked.

Our approach and model have been designed to comply with the methodological guidelines issued by the French government's Major Hazards Institute [3]. The information entered by mayors and local managers is built into a coherent LEP describing the operations to be implemented for each defined crisis scenario. Using the resulting database, OSIRIS-Inondation automatically creates a structured, multi-chapter hypertext document and generates a pdf version for dissemination to the stakeholders and local managers concerned. This document includes maps, tables, illustrations (photos) and, as appendices, reference documents selected by the tool's manager (regulations, directories, etc.).

\subsection{Interface with hydrological forecast and management support}

In the crisis management phase, OSIRIS-Inondation can be interfaced automatically or manually to an official/local hydrological forecast (i.e. on the closest reference gauge). OSIRIS-Inondation is able to combine an Internetbased bulletin with one or more flood scenarios, calculate and schedule the corresponding action plan (i.e. by taking into account the duration of each step) and provide suggestions on how to perform these actions.

The tool's objective is to provide the decision-maker with the information that he/she requires at any given moment. For any given expected or forecast crisis situation, the emergency services manager can thus visualize the number of people affected, the actions required and the stakeholders to be contacted.

OSIRIS was originally designed to enable town councils to manage crisis situations. It is now also intended to facilitate information exchange between the different stakeholders in the crisis (the mayor, the emergency services, transport network and utilities managers, etc.) and the various territorial levels of authority. Improving information exchange before and during a crisis would help to optimize resource sharing and action implementation (especially in major crises). Moreover, major crises do not stop at administrative boundaries; they affect areas outside the locality, in terms of both threatened facilities and the need to bring in personnel and equipment from outside the territorial entity in question (requisition of army units, helicopter airlifts, provision of pumping equipment, etc.).

Hence, most of the preventive actions for countering hazards and protecting local inhabitants are dealt with on a broader scale: neighbouring communes, the county, the region or even nationwide. Implementation of the OSIRIS platform can improve coordination on this scale.

For the Meuse, a unified, automatic representation of flood scenarios has been made possible by interfacing OSIRIS to a unique hydraulic forecast that covers the whole river basin (MOISE, from the engineering firm BCEOM - see. Fig. 1). 


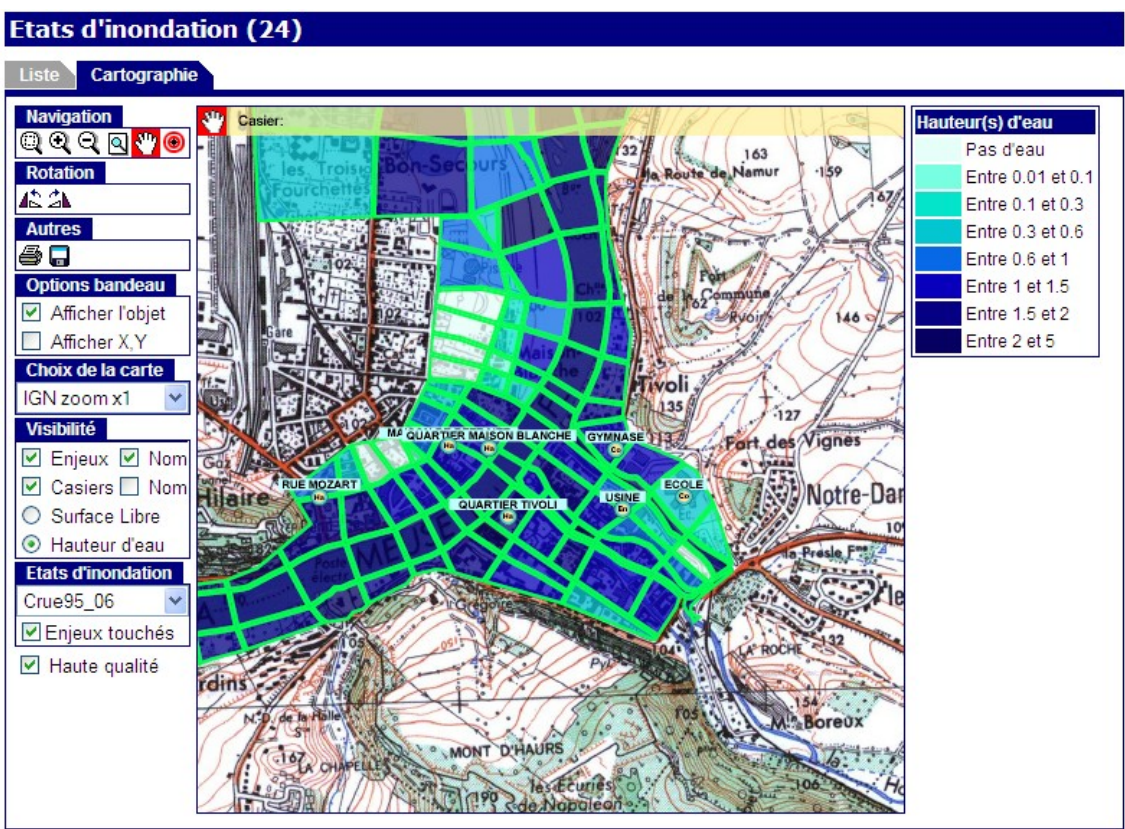

Figure 1: Water levels in hydraulic sectors and detection of affected facilities in OSIRIS.

\subsection{Technical characteristics and dissemination}

On the computing level, OSIRIS-Inondation is based on open-source architecture and Internet technologies (PHP/MySQL) which enable the tool to be used in local or remote-access mode. It is also distributed over the Internet (www.osirisinondation.fr). Thanks to a small, integrated mapping module with basic geographical information system (GIS) functions, the tool is simple to use and quick to learn - notably for non-specialist users, such as municipal employees.

The OSIRIS-Inondation model has been designed to adapt to various local configurations and to the amount and nature of the data available on site. In addition to the standard kernel, OSIRIS-Inondation features a number of configurable options, such as definition of facilities vulnerability depending on the availability and precision of local water levels and the facilities' topography.

Special attention has been paid to promoting and disseminating the tool. Training and promotional material for municipal and state authorities has been created. Furthermore, a progressive training cycle has been set up to help communes adopt the LEP approach and implement it on the OSIRIS platform. 


\section{Impact of flooding on the road network and facility accessibility}

\subsection{Introducing indirect vulnerability}

Since flooding will potentially have many different impacts on a territory, various typologies of vulnerability have been suggested in the literature [4]. In a context of crisis preparation and management support, the main issues relate to the direct and indirect consequences of flooding on the territory and human activities and thus ways of anticipating and limiting these impacts (notably thanks to better forecasting) and optimizing action plans.

Furthermore, the action plan calculated by OSIRIS at a given moment during the crisis does not solve all the problems involved in real-time crisis management in the field: unexpected events, changes in resource availability, communication problems, the high number of stakeholders, etc.

In particular, damage to transport and telecommunications networks and utilities can exert a domino effect: a major initial impact on day-to-day activities, followed by disrupted implementation of emergency action plans [5]. As part of the ACCEL research project, we are specifically studying the various impacts of flooding on road networks and facility accessibility during a crisis.

\subsection{Definition of the methodology and the resulting decision support services}

Decision support in a complex situation means that the operator has to understand and follow the advice given; this presupposes at least some interaction between the user and the system and thus a user mode that matches the operational context. This is why we envision decision support as a set of $a$ la carte services (a tool box, in other words) with which the user can evaluate and compare several situations and alternative scenarios during the crisis preparation or management phase.

To this end, we formulated our initial objectives for the transport management services to be incorporated into the action plan:

1. Detection of roads closed or disrupted by a stationary flood state or a sequential scenario by comparing the hydraulic layer (water levels) with the road network layer. In a real-time crisis management context, it is essential to be able to interactively indicate which sections of the road network are indeed cut off.

2. Detection of the action plan steps likely to be affected by the network disruption: for each starting point/destination pair (type of emergency service/facility to be accessed), we look at whether or not the default itinerary (calculated with a conventional algorithm - see §4.3.1) involves a road that has been cut off or disrupted. If this is the case, OSIRIS will calculate an alternative itinerary and provide a route map.

3. Calculation of an optimized itinerary (based on the journey time) between a starting point and a set of destinations, by taking into account 
flooded roads (see $§ 4.3 .3$ ). This service meets the needs of emergency services managers who must perform a given number of operations in their zone with limited resources (vehicles, personnel, equipment, etc.) and under time pressure (due to rising water levels).

4. Calculation and display of the accessibility and service maps (see \$4.3.2): when the location of the interventional resource is known, the accessibility map represents the time needed to reach any point in the area (e.g. facilities to which access is required) from the nearest emergency service centre for a given flood state. The service map defines the interventional area for each emergency centre, i.e. the mapped area which can be serviced in the shortest delay (see Fig. 3)

\subsection{Identification of data and parameters to be taken into account}

To develop these decision support services, we identified four major categories of data to be acquired and integrated:

1. flood maps, including the spatial boundaries of the flooded area and the water heights, for a range of representative scenarios.

2. road network maps, represented as a $2 \mathrm{D}$ graph (for calculation of the itinerary) with an altitude profile along each section (for the detection of low points and road closures), supplemented if possible by traffic flow data (average speeds depending on the type of zone, traffic hypotheses, etc.).

3. data on crisis management and the action plans developed by the local authorities: these notably concern the identification of vulnerable facilities, the preventive or curative actions to be implemented in the event of a crisis and the various stakeholders and resources used to perform these actions.

4. human and sociological factors (notably how victims react in a crisis) likely to play a decisive role in action plan implementation.

The OSIRIS operational model already includes data on hazards and facilities/actions and exploits the latter to analyze direct vulnerability (damage to designated facilities). Its two new features concern (i) the introduction of a topological layer representing a given transport/communication network and (ii) a set of criteria for defining the notion of accessibility to network nodes. For completeness' sake, we listed "human and sociological factors" above but these are more difficult to model. These will be taken into account in a subsequent phase, when we study the question of evacuations in more detail.

\section{Implementation and testing of the services in the Compiègne urban area}

\subsection{Data acquisition and limitations in terms of accuracy}

The implementation of the services listed in $\$ 3.2$ involved preparation and integration of the accurate digital data presented in $\S 3.3$. This data is not directly 
available and ready to use for the Compiègne urban area (as it is also the case for the great majority of French towns analyzed with OSIRIS) and thus required compilation, pre-processing and even some in situ acquisition work drawing on various local, regional and national datasets.

The Compiègne urban area is exposed to a threat of flooding by the River Oise and its affluent the River Aisne. We tested our method using (i) the road network provided by the French National Geographic Institute's (IGN) BD TOPO database, (ii) flood scenarios produced by hydraulic modelling and local studies, (iii) the European Environment Agency's CORINE Land Cover database (which gives an idea of road traffic fluidity in urban areas) and (iv) a database on the region's strategic facilities (prepared by the Compiègne fire service SDIS60).

In terms of the dataset's quality and adequacy in relation to our objectives, we found that the georeferenced $\mathrm{x}-\mathrm{y}$ map data was generally satisfactory but that $\mathrm{z}$ axis data (i.e. altitude above sea level) was not accurate enough for flooding simulations; the latter requires an accuracy of at least $10 \mathrm{~cm}$ in terms of both ground altitudes and water levels. An acquisition session with a GPS altimeter has been carried out to refine the height data for the designated facilities but this method is not effective enough to be used systematically on a wider scale.

We are following up several potential ways of improving the accuracy of the data by focusing on available large-scale, regularly updated and (if possible) royalty-free databases. In this context, there are some interesting new initiatives in the development of international, community-based web databases, such as OpenStreetMap evaluated by Touya [6].

\subsection{Implementation of the services using ArcGIS and Network-Analyst}

Use of a GIS means that heterogeneous data can be converted into coherent layers, which are then compared and tested in complex spatiotemporal processing steps. This is how the initial services for diagnosing road accessibility (as defined in §3.2) were implemented with ArcGIS and its Network Analyst module within the boundaries of the Compiègne urban area.

Cut-off roads are detected by querying the database and comparing the altitude profile along the itinerary with the water level calculated by numerical simulation (see Fig. 2). Once the road network from the BD TOPO database has been converted into ArcGIS format, the Network Analyst module's functions can be used to calculate an optimized route between two or more strategic points in the designated territory (e.g. between the Compiègne Fire Station or Police Station and the facilities at which action would be necessary in the event of a crisis) by taking account of roads closed or disrupted by flooding.

Lastly, by using relatively simple criteria such as the average road speed (given the water levels and urban density) and the length of unaffected sections, the system generates (i) a service map for all the target facilities from one or more emergency centres and (ii) optimized routes for accessing the facilities by road. 


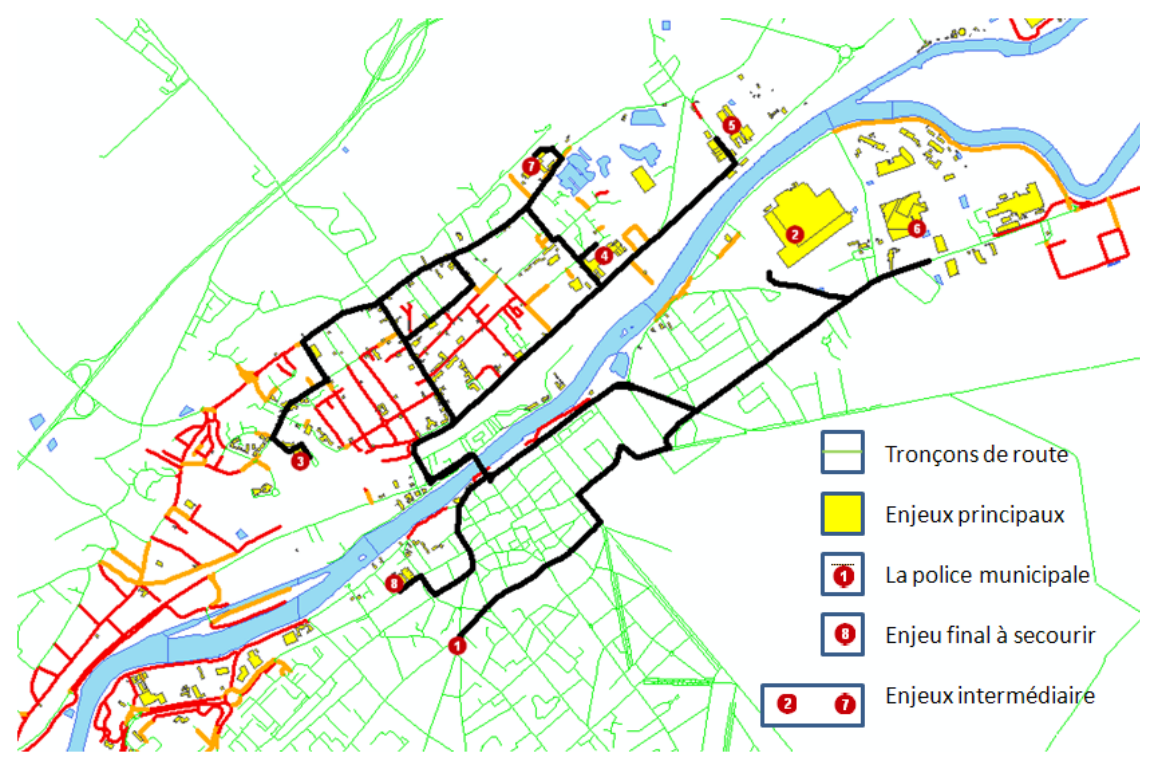

Figure 2: Use of ArcGIS to identify roads that are likely to be cut off or flooded (in red) and to generate optimized access routes (in black).

\subsection{Development of a stand-alone module and its integration into the OSIRIS platform}

A tool like ArcGIS is useful for obtaining results rapidly but is a complex, costly tool designed for specialists. ArcGIS is not intended to be operational and widely disseminated like OSIRIS. Hence, in parallel with the above-mentioned work, we developed a prototype stand-alone software module for providing decision support services based on the same underlying data and which could ultimately be integrated more easily into our OSIRIS platform.

The resulting $\mathrm{C}++$ module can directly import the vector layers describing the transport networks from IGN digital maps (BD TOPO, BD CARTO, Route 120, Route 500 , etc.) or convert the OpenStreetMap database on the fly into a workable format and then evaluate the extent of network disruption in various flood scenarios. In the prototype, the water surface during flooding is considered to be flat but not horizontal; this approximation is valid for areas of a few dozen square kilometres. Comparison of these data does not yet take account of the presence of dikes and other protective infrastructures. The tool's functions are described below.

\subsubsection{Calculation of the optimal itinerary}

For each flood scenario, it is possible to calculate in real time a route between an emergency centre and a facility. To do so, a topological representation of the transport network is calculated from the databases. Each junction is represented by a vertex and each road section is represented by a directed edge. Two-way 
streets are represented by an edge in each direction. Each edge is assigned with a travel time which depends on the depth of the water for that road section and, potentially, the type of vehicle. The quickest route between the service centre and the facility is then calculated by using an $A^{*}$ algorithm [7] - an improvement of Dijkstra's algorithm which decreases calculation times by first testing the most likely routes on the basis of a heuristic function for estimating the remaining travel time at each point of the journey. The route is considered to be optimal as long as the output of the heuristic function is always below the true remaining travel time. Choice of the heuristic function is quite tricky. The user can modify this choice but the default value is:

$$
\operatorname{Time}(A B)=\frac{A B}{2 \bar{v}}
$$

where $\bar{v}$ is the average travelling speed for the road network (i.e. the total length of the road network divided by the total time needed to travel over it). This choice is only valid for urban zones with similar travel speeds, which is the case here.

\subsubsection{Service and accessibility maps}

The software can also draw service maps which represent (for a given flood scenario) the geographical zone which is best serviced by each emergency centre, i.e. the set of points where are more quickly accessible from a given centre than from all the other centres in the general area. Figure 3(b) represents the service map for the Compiègne urban area during the 1993 flood. It takes account of the true positions of the emergency centres (each coloured zone is served by a different emergency centre).

Moreover, in order to identify the areas that are the most difficult for the emergency services to access, it is possible to draw an accessibility map. In Figure 3(a), for example, the areas depicted in light colours (white to yellow) are most easily accessible by the emergency services. The red zones are the most difficult to access and have to be managed with specific hazard prevention and safety instruments.

In order to generate service and accessibility maps, it is necessary to determine the minimum access time from an emergency centre to each point of the road network. To this end, Dijkstra's algorithm is applied once for every emergency centre and the journey times are compared in order to identify the nearest centre.

For points outside the network, interpolation is performed by using the three closest network vertex and applying a penalty function increasing with the distance to the nearest road section. Map generation then involves analyzing a large number of points (about one million). In order to perform these analyses on a time scale that is compatible with an operational response, the topological representation of the road network is improved by using a k-dimensional tree [8]. This decreases the complexity of the search for the nearest vertex in the worst case scenario from $O(n)$ to $O(\sqrt{n})$, where $\mathrm{n}$ is the number of network vertex. 


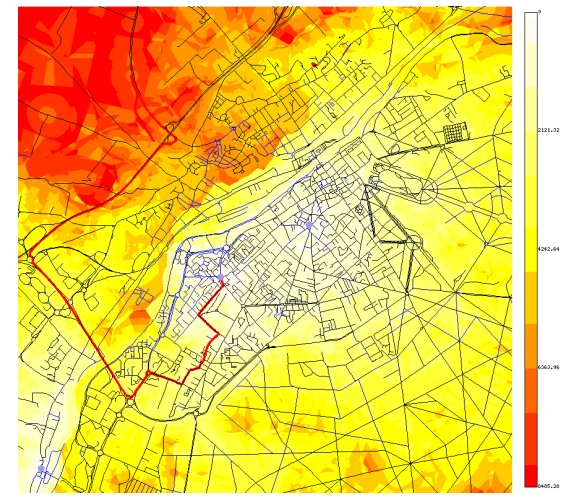

(a)

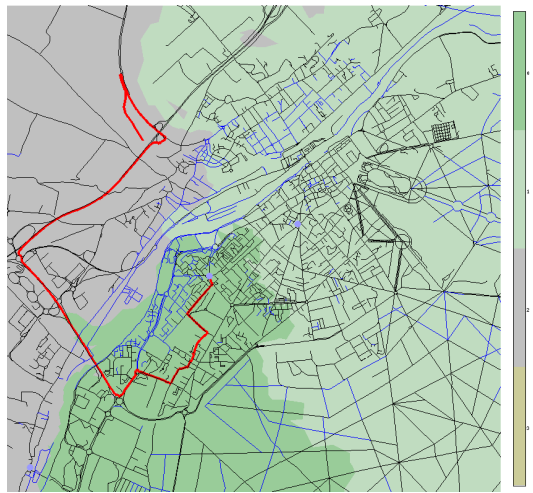

(b)

Figure 3: An accessibility map (a) and a service map (b) for the 1993 Compiègne flood.

\subsubsection{Optimization of emergency service action plans}

Once we have determined the optimal itinerary from an emergency centre to a facility, we can improve the action plan suggested by OSIRIS for each resource involved in the crisis management by indicating the optimal order in which actions should be performed, in order to avoid closed roads and execute all the planned operations as soon as possible.

This problem is analogous to the travelling salesman problem, the apparent simplicity of which masks the complexity of its solution. This is an NP-hard problem: once there are more than a dozen or so facilities, it is not possible to explore all possible routes and one must therefore adopt an approximation algorithm. The software therefore automatically switches from a complete solution to a heuristic algorithm as soon as the number of facilities exceeds 12 . The heuristic approach consists in first determining the optimal routes from each facility to all the others by using an $\mathrm{O}\left(\mathrm{n}^{3}\right)$ complexity Floyd-Warshall algorithm (which is reasonable if there are no more than a few hundred facilities).

In a simplified network consisting only of the facilities and the initial positions of the emergency teams (in which each edge is assigned with the journey time between two vertex), once can then apply an evolutionary ant colony algorithm [9]. The later provides a workable (but not necessarily optimal) action plan in a few tenths of a second. However, experience shows that the generated plan does correspond to the optimal plan in our system.

For each iteration, 800 ants are sent onto the network to seek the optimal route. The ants move randomly along each route until they have covered the network but must never pass through the same vertex twice (although this does not mean that they cannot travel through a facility more than once). When they arrive at their target destination, the ant retraces its steps while depositing on each edge a quantity of pheromones that is inversely proportional to the length of the journey. In subsequent iterations, the ants prefer to pass through edges which 
have accumulated the most pheromone, according to the following probability function:

$$
p i=\frac{\tau_{i}^{\alpha} \eta_{i}^{\beta}}{\sum_{j} \tau_{j}{ }^{\alpha} \eta_{j}{ }^{\beta}}
$$

where $\tau_{i}$ is the density of pheromone on an edge $i, \eta_{i}$ is the attractiveness of the edge $i$ (the reciprocal of its weighting), $\alpha$ is an attractiveness parameter (which, after testing, we set to a value of 1 ) and $\beta$ is a visibility parameter (set to a value of 2). Furthermore, after each iteration, the pheromone evaporates from the edges according to the following equation:

$$
\tau_{i}(t+1)=(1-\rho) \tau_{i}(t)
$$

where $\rho=0.2$. The $\alpha, \beta$, and $\rho$ parameters are chosen after successive trials.

Convergence of the algorithm can then be observed by plotting the shortest journey time against the number of iterations.

\section{Conclusions and perspectives}

In the present work, we have presented an operational decision support tool for flood crisis preparation and management (OSIRIS-Inondation) and the latter's shortcomings in terms of action plan implementation when faced with unexpected events and a flood's indirect consequences on human activities. One of the major shortcomings relates to the impact of floods on the transport networks and the problems that this poses in terms of access to facilities during a flood episode.

The novel software functions identified in this analysis and then subsequently developed will help improve the OSIRIS decision support services in terms of action plan optimization and management during both the crisis preparation phase (analysis and comparison of scenarios) and management phase.

Our methodology for defining and testing accessibility was based essentially on spatial and temporal criteria (distance, journey time, road closures, etc.). It must now be supplemented with other criteria which are more specifically related to the facilities in question (local water height, number of storeys, relative importance, number of people with reduced mobility, the cost of damage, etc.) and the crisis management resources (the types of vehicles, personnel and skills that can be deployed, etc.). The methodology must also take greater account of the dynamics of journeys and traffic congestion modelling [10].

Furthermore, this work will serve as a basis for further studying the question of mass evacuations in major crises - notably as part of the EU funded THESEUS project (FP7-Environment), which deals with the vulnerability of coastal zones and the development of adaptation strategies in a context of climate change. 


\section{Acknowledgement}

We duly acknowledge the support of Conseil Régional de Picardie (France) through the ACCEL project (2007-2010).

\section{References}

[1] Demoraes F., D’Ercole R., Metzger P., Souris M., Enjeux, mobilité, accessibilité et risques. Revue internationale de Géomatique, 16(3-4), Information géographique et gestion des risques, Lavoisier, Paris, pp 435 456, 2006.

[2] van Zuilekom K., van Maarseveen M., van der Doel M., A Decision Support System for Preventive Evacuation of People. Geo-information for Disaster management, Springer-Verlag, pp 229-253, 2005.

[3] DDSC ministère de l'Intérieur, IRMA. Plan communal de sauvegarde, guide d'élaboration pratique, ministère de l'Intérieur, Paris, 2006.

[4] Barroca B., Bernadara P., Mouchel J.M., Hubert G., Indicators for identification of urban flooding vulnerability, Natural Hazards and Earth System Sciences, 6, pp 553-561, 2006.

[5] MEDD, ministère de l'Ecologie et du Développement Durable. Réduire la vulnérabilité des réseaux urbains aux inondations. MEDD, 116 p, 2005.

[6] Touya G., Girres J.F., Première évaluation de la qualité des données libres d'OpenStreetMap en France. Proc. of SAGEO 2009 colloque, Paris, 2009.

[7] Dechter R., Pearl J., Generalized best-first strategies and the optimality of A*. Journal of the ACM, 32(3), pp 505-536, June 1995.

[8] Bentley J.L., Multidimensional divide and conquer. Communications of the $A C M, 23,1980$, pp 214-229

[9] Dorigo M., Gambardella L.M., Ant colony system: a cooperative learning approach to the travelling salesman problem. IEEE transactions on evolutionary computation, 1(1), pp 53-66, 1997.

[10] Stepanov A., MacGregor Smith J., Multi-objective evacuation routing in transportation networks. European Journal of Operational Research, 198, pp 435-446, 2009. 